\title{
COMPARING THE PERFORMANCE OF PREDICTIVE MODELS CONSTRUCTED USING THE TECHNIQUES OF FEED-FORWORD AND GENERALIZED REGRESSION NEURAL NETWORKS
}

\author{
Adeleke Raheem Ajiboye, Ruzaini Abdullah-Arshah, Hongwu Qin, Jamila Abdul-Hadi \\ Faculty of Computer Systems \& Software Engineering, Universiti Malaysia Pahang, \\ 26300 Gambang, Pahang, Malaysia \\ Email: ajibraheem@live.com \\ Phone: +601116214234
}

\begin{abstract}
Artificial Neural Network (ANNs) is an efficient machine learning method that can be used to fits model from data for prediction purposes. It is capable of modelling the class prediction as a nonlinear combination of the inputs. However, a number of factors may affect the accuracy of the model created using this approach. The choice of network type and how the network is optimally configured plays important role in the performance of a predictive model created using neural network techniques. This paper compares the accuracy of two typical neural network techniques used for creating a predictive model. The techniques are feed-forward neural network and the generalized regression networks. The model created using both techniques are evaluated for correctness. The resulting outputs show that, the Generalized Regression Neural Network (GRNN) consistently produces a more accurate result. Findings further show that, the fitting of the network predictive model using the technique of Feed-forward Neural Network (FNN) records error value of 1.086 higher than the generalized regression network.
\end{abstract}

Keywords: Feed-forward network, generalized regression, machine learning, prediction

\section{INTRODUCTION}

Prediction model is the task of building a model of the target variable as a function of the explanatory variables. A neural network is a machine learning method that is designed to model the way in which the brain performs a particular task or function of interest; the network is usually implemented by using electronic components (Haykin, 2009). Neural networks are generally good at fitting functions for prediction purposes. Some types of neural network techniques that can be used to fit such network models include: feed-forward, generalized regression, Hopfield, recurrent network and others.

Creating a predictive model is mostly performed using the feed-forward or generalized regression networks, there are some other techniques too. Prior to using any of these methods for model building, there are some tasks that must be performed on the data. There is need to pre-process the data to be trained, this is to ensure that, the data are in a suitable format for training. Also, there may be need to normalize the data, and in the process, string datasets are coded to numeric values of choice. Several methods 
are known to be appropriate for making such normalization as listed in (Kantardzic, 2011), those methods listed as effective normalization techniques include: decimal scaling, min-max and standard deviation. In the course of creating a network model, the training data iterates for a specified number of epochs and subsequently converges; the relationship between the input attributes and the target attribute is thus established. The developed network model can then be used to generate a prediction of the target attribute from the new untrained attributes. This is to allow prediction of unknown information by making use of the already known data.

While Feed-Forward Neural Network (FNN) technique requires back-propagation algorithm for training, Generalized Regression Neural Network (GRNN) technique does not require this. Hence, the generalized regression neural network does not face the frequently encountered local minima problem noticed in feed-forward back propagation applications (Cigizoglu, 2005). Both techniques have been widely used for problem solving and they are well reported for model construction.

A number of neural network techniques are known for fitting of models; however, the focus of this study is to compare the network models created using these approaches, with a view to determining their level of accuracy. Specifically, this study aimed at investigating the accuracy of the network prediction model created using the techniques of feed-forward and generalised regression networks. Findings from this paper would guide on the choice of these neural network techniques for the creation of network model for prediction purposes.

The rest of this paper is organized as follows: In the next section, a brief overview of neural network is presented. This is followed by some related work reported in the literature. Next to this section is a discussion on the materials used and the presentation of the proposed method. This is followed by the discussion of the results, and findings of this study. The conclusion of this paper is presented next to the discussion of results.

\section{AN OVERVIEW OF ARTIFICIAL NEURAL NETWORKS}

An artificial neural network is a type of artificial intelligence that attempts to mimic the way the human brain processes and stores information (Taylor, 2006). A neural network is a biologically inspired non-linear parallel computing paradigm for information processing. It has exploratory analysis, having a distinct ordering among the sets of neurons arranged as input and output layers with zero or more processing hidden layers that are interconnected by signal channels and fine-tuned by a training algorithm (Chattamvelli, 2009).

Neural network derives its computing power through its massively parallel distributed structure and its ability to learn, which make it possible for it to generalize. Generalization refers to the neural network's production of reasonable outputs for inputs not encountered during the learning process (Haykin, 2009). These two information processing capabilities make it possible for neural networks to find good approximate solutions to complex and large-scale problems that are intractable.

The techniques of Artificial Neural Networks (ANNs) for model construction are well-established modelling method; it uses neurons to process data. A neuron is an information-processing unit that is fundamental to the operation of a neural network. It is the basic building block for all types of neural networks. In neural networks, the neurons are connected by links, and each link has a numerical weight associated with it. Weight are the basic means of long-term memory in ANNs (Negnevitsky, 2011). The capability of a single neuron is very limited; a number of neurons can be connected in 
order to boost the processing power of the network. Figure 1, illustrates a typical neuron.

The neural model represented in Figure 1, includes an externally applied bias, denoted by $b_{k}$. The bias $b_{k}$ has the effect of increasing or lowering the net input of the activation function, depending on whether it is positive or negative, respectively. In mathematical terms, the neuron $k$ depicted in Figure 1, can be described by writing the pair of equations (1) and (2):

$$
u_{k}=\sum_{j=1}^{m} w_{k j} x_{j}
$$

and

$$
y_{k}=\varphi\left(u_{k}+b_{k}\right)
$$

where $x_{1}, x_{2}, \ldots, x_{m}$ are the input signals; $\mathrm{w}_{k 1}, \mathrm{w}_{k 2}, \ldots, \mathrm{w}_{k m}$ are the respective synaptic weights of neuron $k ; ; b k$ is the bias; $\varphi(\cdot)$ is the activation function; and $y k$ is the output signal of the neuron

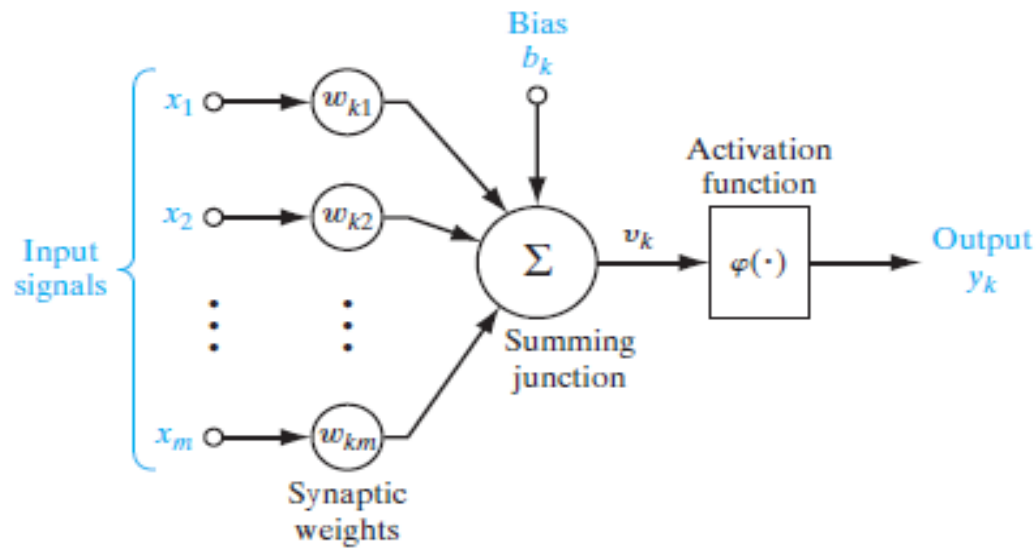

Figure 1. The model of a neuron adapted from (Haykin, 2009).

To propagate error signal involves moving from the output layer and in the backward direction towards the hidden layer. The error signal at the output of neuron $k$ at iteration $p$ as defined in (Negnevitsky, 2011) is:

$$
\mathrm{e}_{\mathrm{k}}(p)=y_{\mathrm{d}, \mathrm{k}}(p)-\mathrm{y}_{\mathrm{k}}(p)
$$

where $y_{\mathrm{d}, \mathrm{k}}(p)$ is the desired output of neuron $k$ at iteration $p$ and $\mathrm{y}_{\mathrm{k}}(p)$ is the network output.

It has been observed that one of the fundamental application problems in computing today is the development of systems that can carry out the rapid, reliable, automatic recognition and classification of complex patterns. Artificial neural networks are naturally suited to solving pattern classification problems through machine learning (Hancock, 2012). Neural networks offer more useful properties and capabilities, some of these are listed in (Haykin, 2009) as : Nonlinearity, Input-Output Mapping, Adaptivity, Evidential Response, Fault Tolerance, Uniformity of Analysis and Design etc. 


\section{RELATED WORK}

Several techniques, especially machine learning, are known for the creation of predictive models; among other methods that can be used to fits model from data, the techniques of neural networks appear to be well reported in the literature. Both FNN and GRNN have been identified as efficient techniques and capable of constructing useful prediction models, a number of these models are reviewed in this section.

The study in (Kutyłowska, 2014), uses the multi-layered perceptron which is a feed-forward neural network to create a model for failure rate prediction. From the engineering point of view, the model trained with the back propagation algorithm, was evaluated to have given an acceptable convergence. The study opined that, the model created can be used for water utility in the future to establish the level of failure frequency and to plan the renovation of the most deteriorated pipes.

Also, a study reported in (Mishra \& Desai, 2006), constructs a predictive model using feed-forward neural network to forecast drought for effective planning and management of natural resources. The paper compares a number of neural networks techniques such as direct multi step, recursive multi step and stochastic model. The paper presented the potentials of the three models to effectively forecast drought over a lead time.

The study in (Pijanowski et al., 2002), uses the technique of FNN to create a land transformation model for forecasting of land use changes. The technique has also been reported for prediction of waste water treatment (Hamed et al., 2004). The technique is also found useful in Chemistry, especially for the molecular classification and pattern recognitions (Burns \& Whitesides, 1993).

Study in (Cigizoglu \& Alp, 2006) uses Generalized Regression Neural Network (GRNN) in the modelling of river sediment yield. Unlike FNN, the generalized regression neural network does not require an iterative training procedure as in back propagation method and the resulting estimations from the developed model were found to be significantly superior to conventional method results. Leung et al. (Leung et al., 2000) developed a model using GRNN for the purpose of forecasting exchange rate and compares its performance with a variety of forecasting techniques and reported that, apart from having a higher degree of prediction accuracy, GRNN performs statistically better than other evaluated models compared in the study for different currencies. GRNN has also been reported to have performed well in prediction to forecast plant disease (Chtioui et al., 1999), and modelling of plasma etching (Kim et al., 2003).

The effectiveness of using neural network for prediction as reported in a review carried out in (Adya \& Collopy, 1998) unveils some capabilities and suitability of both techniques. The proposed study further reveals the strength of these techniques and findings of this study shows that, both techniques are well suitable for prediction and can be relied upon.

\section{MATERIAL AND METHODS}

The data used for the implementation of the models created using both network techniques is discussed in this section. The study explores a cross-country database for sector investment and capital, retrieved from the open data repository of the World Bank, the url of the data source is represented at the end of reference listing in this paper. The study aimed at modelling the capital investments, which is here referred to as input attributes and the Gross Domestic Product (GDP), which we refers to the target attribute. The 570 data sets explored comprised of 4 input attributes and 1 target 
attribute. Experiments were conducted to create prediction models based on the two techniques FNN and GRNN. The data are pre-processed and they are put into the proper format for training.

\section{Experimental procedures}

Experiment I. The first model is created using FNN technique and trained with 400 data sets; the same size of data is used to create the second model using GRNN technique. The feed-forward neural network is trained using back propagation algorithm. The basic configuration of FNN during the experiment is represented in Table 1, while other settings are left at their defaults and its structure is shown in Figure 2.

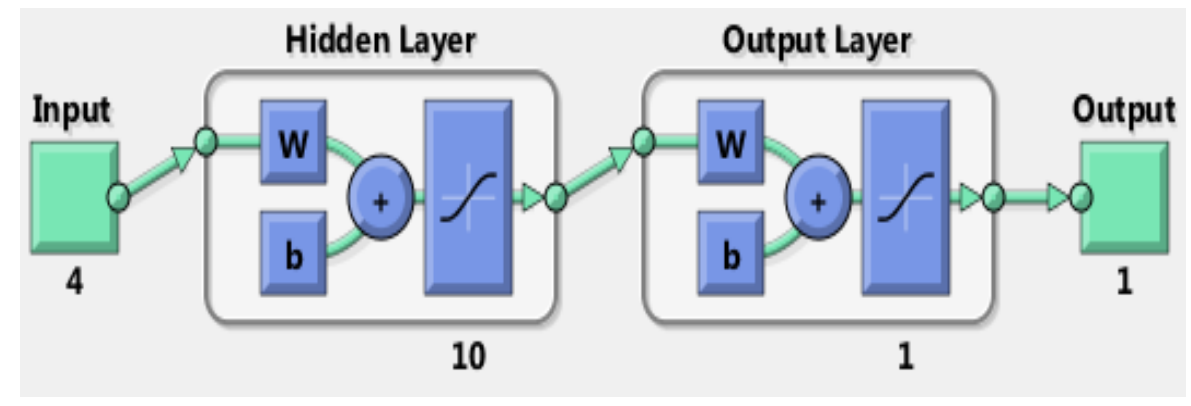

Figure 2. Feed-forward neural network architecture

Table 1: FNN configuration

\begin{tabular}{|c|c|}
\hline Algorithm & $\begin{array}{l}\text { Data Division: Random } \\
\text { Training: Back propagation }\end{array}$ \\
\hline Network properties & $\begin{array}{l}\text { Network type: Feed-forward BP } \\
\text { Performance: Mean Square Error } \\
\text { Number of Neurons: } 10\end{array}$ \\
\hline Network Parameters & $\begin{array}{l}\text { Epochs: } 750 \\
\text { Goal: } 0 \\
\text { Min grad: } 1 \mathrm{e}-7 \\
\text { Validation checks: } 8 \\
\text { Mu: } 0.001\end{array}$ \\
\hline
\end{tabular}

Also, the network model created through the generalized regression technique is represented in Figure 3. It uses purelin transfer function towards the output layer. The network model is created using the same predictors and the target data.

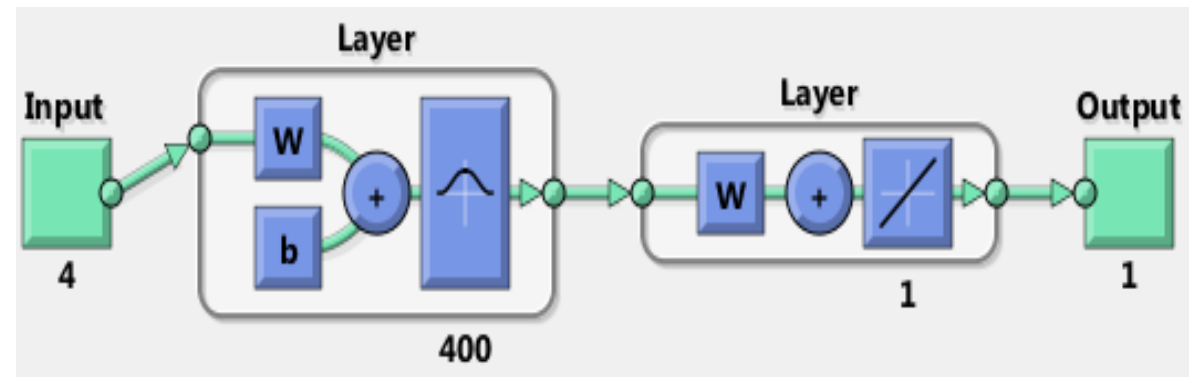


Figure 3. Generalized regression neural network architecture

This concludes the fittings of the two network predictive models from data, using the network type: feed-forward and generalized regression.

Experiment II. Each predictive model created in the previous experiment is evaluated using a new set of 170 data. In order to establish the accuracy of each model, the error associated with each model is computed and comparison is made. The Mean Absolute Error (MAE) computed for the models created using both techniques is based on Equation (3):

$$
\mathrm{MAE}=\frac{\left|p_{1}-t_{1}\right|+\ldots .+\left|p_{n}-t_{n}\right|}{n}
$$

To compute the MAE, the required parameters are the predicted outputs and the target data. The network predicted output values are $p_{1}, p_{2}, \ldots ., p_{n}$; the target values are $t_{1}$, $t_{2} \ldots, t_{n}$ while $n$ is the number of sample dataset. The errors computed for each network model are represented in Table 2.

\section{RESULTS AND DISCUSSION}

In this section, the results of comparing the accuracy of the network model created using the techniques of feed-forward and generalized regression is illustrated and discussed. The training performance of the prediction models created using the FNN technique is represented in Figure 4. Specifically, the graph shows the training, validation and testing data during the training process. It can be seen from the figure that, the best performance is recorded at iteration 49 , before it finally converges at epochs 55 to avoid over-fitting. Looking at the graph further, the line that represents the validation and test, exhibit some similarities and at the time of convergence, no sharp increase in error is noticed in the validation data.

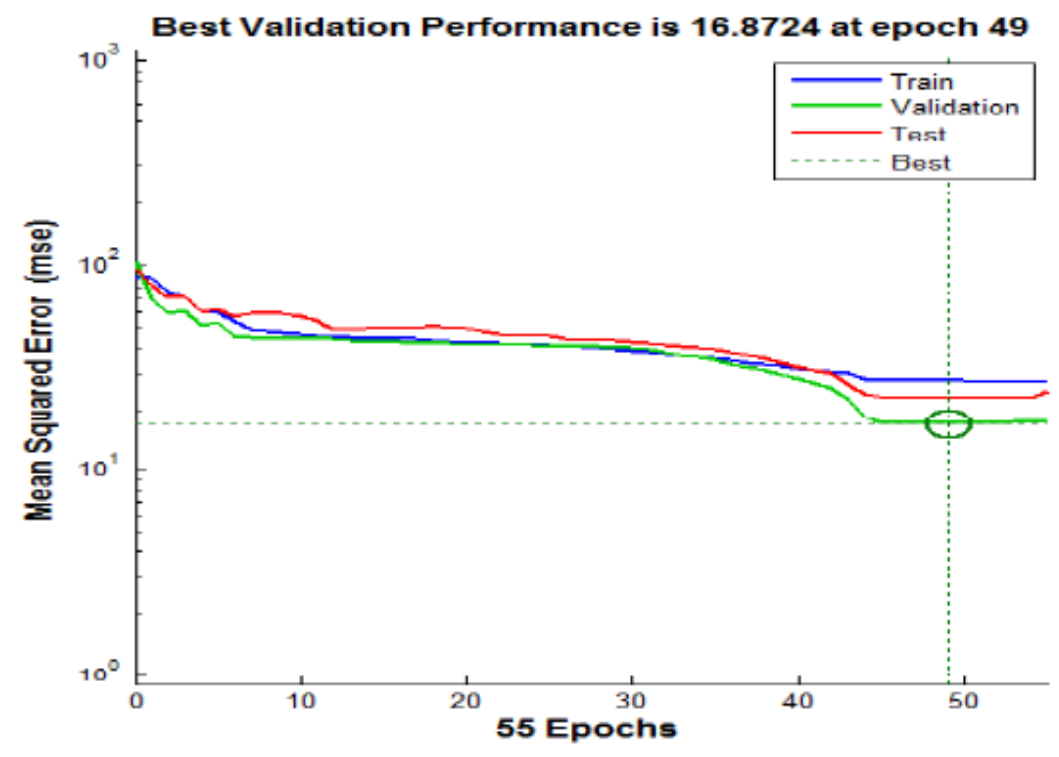

Figure 4. FNN training performance 
As said earlier, in order to determine the accuracy of the models created through both techniques, each model is simulated with sets of new data. Simulating a network model only requires the input data (predictors). This produces a predicted output for each network model. Such evaluation involves finding the difference between the predicted outputs and the target outputs. Also, in order to be more specific with the error values associated with each network model, the MAE computed in respect of each structure is numerically represented as shown in Table 2 . The choice of error measurement for the simulated results in this paper is MAE; as measuring of the numeric prediction using MAE does not tend to exaggerate the effect of outliers; this is because, all sizes of errors are treated evenly according to their magnitude (Witten et al., 2011).

It can also be seen from Table 2 that, the generalized regression network gives a relatively better accurate result.

Table 2: The errors associated with the network models created from the first data sets

\begin{tabular}{|l|l|l|l|}
\hline $\begin{array}{l}\text { Neural network } \\
\text { technique }\end{array}$ & $\begin{array}{l}\text { Size of data used for } \\
\text { model construction }\end{array}$ & $\begin{array}{l}\text { Untrained data used } \\
\text { for simulation }\end{array}$ & Error \\
\hline FNN & 400 & 170 & 6.879 \\
\hline GRNN & 400 & 170 & 5.793 \\
\hline
\end{tabular}

\section{CONCLUSION}

In this paper, two network types, feed-forward and generalized regression neural networks are implemented to fits predictive model from data. The network models are created using Matlab Software. The public dataset explored in this study experiment with 570. Since the accuracy can be expressed in terms of error, the mean absolute error associated with the model created based on each technique is computed. The comparisons of these errors show that, the generalized regression neural network has lower error and found to perform much better.

The comparisons made in this study reveal the effectiveness of the models created through both techniques. Although, both techniques are suitable for creating prediction models and the two techniques can reliably be used to make useful estimations. However, the findings from this research further shows that, the GRNN is more accurate and can effectively compete with other techniques that are well known for creation of predictive models. 


\section{REFERENCES}

Adya, M., \& Collopy, F. (1998). How effective are neural networks at forecasting and prediction? A review and evaluation. J. Forecasting, 17, 481-495.

Burns, J. A., \& Whitesides, G. M. (1993). Feed-forward neural networks in chemistry: mathematical systems for classification and pattern recognition. Chemical Reviews, 93(8), 2583-2601.

Chattamvelli, R. (2009). Data Mining Methods. Oxford U.K.: Alpha Science.

Chtioui, Y., Panigrahi, S., \& Francl, L. (1999). A generalized regression neural network and its application for leaf wetness prediction to forecast plant disease. Chemometrics and Intelligent Laboratory Systems, 48(1), 47-58.

Cigizoglu, H. K. (2005). Application of generalized regression neural networks to intermittent flow forecasting and estimation. Journal of Hydrologic Engineering, $10(4), 336-341$.

Cigizoglu, H. K., \& Alp, M. (2006). Generalized regression neural network in modelling river sediment yield. Advances in Engineering Software, 37(2), 63-68.

Hamed, M. M., Khalafallah, M. G., \& Hassanien, E. A. (2004). Prediction of waste water treatment plant performance using artificial neural networks. Environmental Modelling \& Software, 19(10), 919-928.

Hancock, M. F. (2012). Practical Data Mining: CRC Press.

Haykin, S. (2009). Neural Networks and Learning Machines (Third ed.). New Jersey: Pearson Prentice Hall.

Kantardzic, M. (2011). DATA MINING: Concepts, Models, Methods, and Algorithms (2nd ed.): John Wiley \& Sons Inc.

Kim, B., Kim, S., \& Kim, K. (2003). Modelling of plasma etching using a generalized regression neural network. Vacuum, 71(4), 497-503.

Kutyłowska, M. (2014). Neural network approach for failure rate prediction. Engineering Failure Analysis.

Leung, M. T., Chen, A.-S., \& Daouk, H. (2000). Forecasting exchange rates using general regression neural networks. Computers \& Operations Research, 27(11), 1093-1110.

Mishra, A., \& Desai, V. (2006). Drought forecasting using feed-forward recursive neural network. Ecological Modelling, 198(1), 127-138.

Negnevitsky, M. (2011). Artificial Intelligence A Guide to Intelligent Systems: Pearson Education Limited.

Pijanowski, B. C., Brown, D. G., Shellito, B. A., \& Manik, G. A. (2002). Using neural networks and GIS to forecast land use changes: a land transformation model. Computers, environment and urban systems, 26(6), 553-575.

Taylor, B. J. (2006). Methods and Procedures for the Verification and Validation of Artificial Neural Networks. USA: Springer Science+Business Media, Inc.

Witten, I. H., Frank, E., \& Hall, M. A. (2011). DATA MINING Practical Machine Learning Tools and Techniques: Morgan Kaufmann.

url for the data source: Retrieved Jan. 5, 2016, from http://go.worldbank.org/K955YO0N00 